\title{
Treatments for fibrosis development and progression: Lessons learned from preclinical models and potential impact on human conditions such as scleroderma, pulmonary fibrosis, hypertrophic scarring and tendinopathies
}

\author{
David A. Hart \\ McCaig Institute for Bone \& Joint, Health University of Calgary, Calgary, Canada \\ Email: hartd@ucalgary.ca \\ Received 15 June 2013; revised 17 July 2013; accepted 25 July 2013 \\ Copyright (C) 2013 David A. Hart. This is an open access article distributed under the Creative Commons Attribution License, which \\ permits unrestricted use, distribution, and reproduction in any medium, provided the original work is properly cited.
}

\begin{abstract}
Progressive fibrosis of a tissue or organ in response to a damaging insult may result in loss of organ function if the acute response is excessive, or a chronic fibrotic response is initiated due to the persistence of the insult. In the author's laboratory over the past several years, a number of preclinical models of fibrosis or fibrogenic responses have been characterized for the effectiveness of various treatment approaches to either prevent or impede fibrosis development and progression to identify commonalities and translatable research directions that could provide insights into human diseases. These have mainly included either chemically induced pulmonary fibrosis models or overt physical injury models in rats, pigs and rabbits. Some preliminary studies in human populations have also been undertaken. The interventions evaluated have included fibrinolytic agents and drugs targeting specific cell populations. The results indicate that some approaches lend themselves to modifying fibrotic reactions in some models and not others, while others may have a more generalized impact on fibrogenic responses due to interference with abnormal cell functions in the injury environment.
\end{abstract}

Keywords: Fibrosis; Chronic Fibrotic Response; Fibrinolytic Agents; Cell Function

\section{INTRODUCTION}

Fibrosis of a tissue or organ can result from a variety of insults that are either direct (e.g. overt physical injury or wounding; skin wound), or indirect as a consequence of a process unrelated to the target tissue (e.g. deposition of autoantibody complexes in the kidney, lung or liver). However, in some situations, there can be a genetic susceptibility of the target tissue, such as the kidney to be susceptible to autoimmune insults [reviewed in 1-4]. Such events may be acute, and potentially resolve, or chronic leading to a progressive loss of function. The pattern of responses to such insults may be influenced by multiple variables such as genetics, previous insults (history), possibly sex and age, as well as co-morbidities. While the spectrum of inciting stimuli which can lead to fibrosis or a fibrotic process are quite diverse (chemicals, microorganisms, autoimmune processes, burns, physical disruptions, loss of function, etc.), the host has a limited set of "tools" to instigate a response to either repair the damage, control the extent of the damage, or try to regenerate the original tissue.

Unfortunately, many of the response patterns do not lead to regeneration, but instead lead to fibrosis with concomitant loss of integrity. In patient populations, many times this loss is accompanied by overt symptoms, but in other conditions the loss is gradual or the outcomes are not obvious in the short term. Therefore, it is critical to develop approaches to interfere with the untoward process rather than block its initiation. In this review, some evidence to suggest that interventions associated with the use of fibrinolytics $+/-$ mast cell stabilizers should be considered to intervene in some disease processes where current options are restricted. Such considerations rely on understanding of the processes underlying the conditions, but also the development of early 
diagnostics and biomarkers for disease activity to assist in the decision making.

\section{FIBRINOLYTIC APPROACHES TO IMPACT FIBRIN DEPOSITION AND FIBROSIS}

\subsection{Rat Bleomycin-Induced Pulmonary Fibrosis Model and Urokinase Effectiveness}

Instillation of the chemical bleomycin into the lungs of rats leads to a progressive fibrosis that is dose dependent [5; reviewed in 6]. It also occurs in some strains of mice and not others, as well as subsets of patients receiving the drug for cancers, presumably due to the genetic presence or absence of enzymes to metabolize the compound [reviewed in 7,8]. As the rat model is well characterized [reviewed in 9,10], and access to rat lungs better for a variety of stimuli, studies were performed with Sprague-Dawley male rats. As fibrosis in this model requires continual deposition of fibrin in reaction to the noxious stimulus, the intervention assessed was urokinase, a plasminogen activator normally found in the lung [reviewed in 6]. The hypothesis was that instillation of urokinase to the affected lungs would result in degradation of the deposited fibrin, thus interfering with the fibrin deposition-fibroblast infiltration-collagen deposition triad contributing to overt and progressive fibrosis. Excessive or chronic expression of procoagulant activity in the lung can lead to fibrin deposition and subsequent fibroblast infiltration and fibrosis [reviewed in 11,12]. Procoagulant activity (e.g. tissue factor, prothrombinases, etc.) can be involved in fibrinogen activation and fibrin deposition in a number of inflammatory conditions with various target organs [11-16].

Rats with bleomycin-induced pulmonary fibrosis tolerated instillation of commercially available human urokinase and evaluation of the extent of collagen deposition using histological assessment methods employing picrosirius red staining of sections of lung tissue and computer-assisted quantitation of collagen under polarized light conditions revealed reproducible induction of fibrosis and partial reversal using urokinase [5]. However, the urokinase was more effective early after induction with bleomycin than later when the early fibrin deposition had become infiltrated with cells and collagen deposited. Furthermore, the urokinase treatment did not disrupt further fibrosis progression so it was a temporary or "stop gap" treatment without long lasting impact. Thus, as a proof of principle, urokinase treatment may be efficacious in situations where there is an acute stimuli provoking the fibrosis, but likely it would not be clinically useful alone when used in isolation for more chronic fibrosis situations such as idiopathic pulmonary fibrosis [6].

\subsection{Rat Model of Cystic Fibrosis and Urokinase Effectiveness}

Building on the above discussed bleomycin model, we turned our attention to a rat model of cystic fibrosis (CF) using Pseudomonas bacteria in agar beads as the inciting agent [17] and once again evaluated the efficacy of urokinase treatment. In this model, a chronic infection arises after instillation of a bolus of bacteria into one lobe of the lung $[17,18]$. The conversion to a chronic infection that mimics human $\mathrm{CF}$ via the change in the bacteria from a planktonic form to a biofilm form mimics what occurs in the lungs of patients with $\mathrm{CF}$.

While the focus of the studies was on fibrinolyticmediated destruction of the fibrosis associated with the chronic infection, an unexpected result was detected. That is, the urokinase was partially effective with regard to its impact on fibrosis [19], but apparently the enzyme can also interact with bacteria to alter their growth [20]. This activity of urokinase was found for a number of bacteria that can infect burn patients [21]. Thus, in this situation of fibrosis associated with bacterial infection, the fibrinolytic approach could be a "double-edged sword" yielding a complicated outcome. In this circumstance, one would likely have to eradicate the microorganisms by another mechanism (e.g. specific antibiotics) prior to attempting to impact the remaining residual fibrosis.

\subsection{Fibrinolytics in Patients with Fibrotic Conditions}

While a number of fibrotic diseases or conditions exist for a variety of target organs (e.g. lung, kidney, liver, skin, etc.), fibrinolytic approaches to affecting the fibrosis have not been well studied. However, some insights have been gained from the study of patients with scleroderma, a disease that leads to thickening and fibrotic responses in the skin $[22,23]$. The phenotype of the skin involvement can vary extensively [reviewed in 22], as well as disease progression, with some patients at risk for cardiovascular events, pulmonary hypertension and pulmonary fibrosis [reviewed in 24]. In many scleroderma patients, the condition is preceded by Raynaud's phenomenon (likely related to an abnormal neuro regulation of vasoconstriction; 25; and others). Scleroderma is considered an autoimmune disease with characteristic autoantibodies present [25; and others].

The potential efficacy of fibrinolytic approaches to impact scleroderma initially arose due to some serendipity, a patient with scleroderma of long duration and significant progression suffered a myocardial event and was entered into a tissue plasminogen activator (tPA) trial using $100 \mathrm{mg}$ of tPA. Almost immediately following 
infusion of the tPA, the patient reported feeling warmth in her feet for the first time in years. Moreover, the patient also exhibited a good response to the tPA in relation to the myocardial event with no further complications [26]. Circulation to the extremities improved for several years following the tPA infusion and the skin involvement appeared to stabilize, but the existing fibrosis did not resolve [26; and discussed in 27].

The efficacy of the tPA in this patient did not address the skin fibrosis, but apparently did impact the dysregulated intravascular fibrin deposition for an extended period of time. Thus, in patients such as the case discussed, some of the progression of the disease is related to intravascular dysregulation which complements the skin thickening.

Subsequent to the above case report [26], a small clinical trial was implemented with a subset of scleroderma patients wirh a treatment regimen of $10 \mathrm{mg}$ tPA (a dose restriction implemented by the providing company to minimize potential bleeding risk associated with higher doses). A cross-over trial format was used and the outcomes related to standard skin scores [27]. Most of the patients did not experience a significant response to this low dose regimen of tPA, but a subset of estrogen-deficient patients did appear to experience a positive response, however, the numbers involved were small.

Subsequently, and sometimes in parallel to those early investigations, a number of studies have been reported which indicate that regulation of fibrinolysis in scleroderma patients is either fairly normal [28] or abnormal [29-31], and that treatment of subsets of scleroderma patients with fibrinolytics such as urokinase or tPA can offer benefit to patients [32-34], but the benefit may not be of long duration [35]. Also, in some patients, antibodies to fibrin bound tPA were detected [36], a finding that could contribute to fibrinolytic abnormalities detected in such patients. In addition, fibroblasts from scleroderma patients have been reported to over express inhibitors of fibrinolysis such as Plasminogen Activator Inhibitor-2 (PAI-2) [37]. or have an altered phenotype in cells from some patients [38]. Recent studies have indicated some genetic variants of the urokinase receptor (UPAR) may present fibrinolytic risk for a subset of patients with scleroderma [39], findings which may explain some of the heterogeneity in patient disease progression and perhaps, responsiveness to fibrinolytic interventions. Therefore, whether the fibrinolytic approach would benefit only a distinct subset of patients with this complex disease to impact the fibrosis and pro-fibrotic environment, must await the outcomes of further studies.

However, additional clinical trials with higher doses of tPA (perhaps 25 and $50 \mathrm{mg}$ ) and possibly multiple exposures, are likely warranted to address the vascular fibrotic/pro-fibrotic environment based on the information gained thus far, but certainly such trials with higher doses would be accompanied by some increased risk for bleeding disorders. As many patients have a progressive disease without many other options, the risk may be acceptable for some patients. Furthermore, as the action of tPA is fibrin dependent, as opposed to urokinase, the tPA approach may offer more benefit to the vascular profibrotic environment, while urokinase, the natural plasminogen activator of the lung may be a better choice for pulmonary actions. Similarly, more studies using urokinase to address the early pulmonary complications in scleroderma are also likely warranted, possibly using the direct instillation of the urokinase into the affected lungs to maximize impact.

Furthermore, as will be discussed below, other approaches may be entertained in subsets of scleroderma patients that could complement the fibrinolysis approach either using tPA or urokinase.

\section{USING MAST CELL STABILIZERS TO INTERFERE WITH FIBROSIS DEVELOPMENT AND FIBROGENIC RESPONSES TO INJURY}

\subsection{The Red Duroc Porcine Model of Abnormal Skin Wound Healing}

The red Duroc pig model of excisional skin wound healing exhibits some features of hypertrophic scarring [4044], but likely it should more accurately be described as a fibrogenic response to injury [45]. Excisional wounds on the dorsum of this breed of pig heal with a hypercontracted phenotype, plus abnormal deposition of collagen (e.g. collagen nodules) and pigmentation, and an abnormal mRNA expression pattern/phenotype (two phases of inflammatory molecule expression). In contrast, healing of similar excisional wounds in animals of the Yorkshire breed proceed with less contraction than what is observed in the red Duroc model. That is, with a single phase of molecular expression, and a pattern not dissimilar to healing in humans. Histologic analysis of scar tissue developing following injury to red Duroc skin revealed elevated numbers of nerves, mast cells and myofibroblasts in red Duroc scars compared to those in Yorkshire animals [46]. This lead to the hypothesis that a nerve-mast cell-myofibroblast axis exists and in the abnormal healing phenotype, and this axis was contributing to the abnormal phenotype in the healing of red Duroc skin wounds [46].

To assess whether stabilizing mast cells and potentially preventing degranulation due to neuropeptide stimulation, red Duroc pigs were provided the mast cell stabilizer ketotifen in a syrup on a biscuit $2 \mathrm{x}$ day, starting at day 0 (time of wounding) or at Day 28 post-wounding. Ketotifen has been used for $>20$ years in both pediatric 
and adult asthma sufferers, and has a fairly well defined safety profile. The doses chosen for the pig models were based on an extrapolation of human doses on a $\mathrm{mg} / \mathrm{kg}$ basis. Controls included feeding red Duroc pigs a syrup without ketotifen, and giving Yorkshire pigs with similar skin wounds similar doses of ketotifen at Day 0 .

The findings indicated that ketotifen treatment starting at Day 0 completely abrogated the abnormal skin wound healing phenotype in the red Duroc animals, but starting treatment at Day 28 post-injury, when re-epithelization was complete did not lead to detectable reversal of the abnormal fibrogenic phenotype [46]. Similarly, stopping treatment of the animals initially treated from Day 0 did not lead to a re-activation of the abnormal healing response. Histologically, ketotifen treatment initiated at Day 0 led to decreased nerves, mast cells and myofibroblasts in the scar tissue. Based on these findings, it was clear that stabilizing mast cells with ketotifen early after wounding was very effective in preventing development of the abnormal fibrogenic response, but the scars healed normally and healing was not compromised. Interestingly, and further to the last point, treatment of injured Yorkshire animals with ketotifen had no detectable effect on the healing outcomes. Therefore, ketotifen prevented development of the abnormal fibrogenic phenotype, but did not compromise normal healing. Thus, this intervenetion can discriminate between normal involvement of nerves, mast cells and myofibroblasts in the healing process of skin, but is somewhat specific for abrogating the abnormal influences. Several factors that remain to be further investigated is the why, where and how the ketotifen is working in the dynamic and complex environment of the wounds. Thus, studies examining ketotifen effectiveness at time points between Day 0 (fully effective) and Day 28 (no effect) post-wounding (e.g. Day $7,14,21)$ may provide additional clues as to when the drug is most effective and further insights into the "how and why" it is effective.

One clue that has arisen relates to cells in the dorsal dermis of the red Duroc pigs. It was found that the behavior of fibroblasts/myofibroblasts derived from the dorsum of the re Duroc animals exhibited differences from those from the dorsum of Yorkshire pigs, or even the ventral skin of the red Durocs [47]. As the ventral skin arise during development separately from the dorsal skin (discussed in [48]), it would appear that endogenous cells at the site of an insult can potentially contribute to the response pattern. However, a direct comparison of wound healing between the dorsal and ventral skin in these pigs has not been reported. Such studies examining the myofibroblasts, nerve properties and mast cells between such sites may provide some additional clues for other disease processes such as in scleroderma which exhibits considerable heterogeneity both within and be- tween patients. Such studies may provide insights into the basis for development and progression of fibrotic processes in specific environments, insights that ultimately could impact the nature of interventions used to negate the processes.

\subsection{Use of Ketotifen in Prevention Joint Contractures Following Joint Injury in a Rabbit Model}

In a significant subpopulation ( $15 \%)$ of individuals experiencing an injury to a joint such as the elbow, the outcome of the repair is less than ideal and the patients suffer from a joint contracture with loss of range of motion that compromises function [discussed in 49-51], with excessive deposition of collagen likely via a fibrogenic response to injury. For those that are severely compromised, the only viable option to help restore some range of motion is surgery to release the affected joint capsule in an attempt to restore healing. Unfortunately, most surgeries result in $\sim 50 \%$ restoration of function at best [reviewed in 52,53], so the intervention is not optimal. Why this subset of patients have an abnormal healing outcome is not known presently, but characterization of affected tissues have provided some insights.

Analysis of tissue from such patients compared to controls at the histologic and molecular levels have indicated a fairly uniform pattern of differences in the affected tissues, including excessive nerves, mast cells and myofibroblasts in affected capsular tissue [49-51]. Thus, there appears to be some parallels between the red Duroc model of abnormal healing and that observed in the human joint contracture situation.

Over the past several years, a rabbit joint injury/joint immobilization model leading to contractures which mimic the pattern of changes observed in patient joint contracture samples has been characterized [54-56]. Many features of this preclinical model parallel those observed in both the human tissue samples and the red Duroc model, namely elevated presence of nerves, mast cells and myofibroblasts [56]. Therefore, this rabbit model is also a likely candidate to assess the efficacy of ketotifen to prevent contracture development.

Treatment of rabbits with a knee injury analogous to injuries suffered by humans to their elbows with ketotifen, was initiated at Day 0 . The ketotifen was injected subcutaneously $2 \mathrm{x} /$ day as it was not possible to effecttively administer the drug orally to the rabbits. The treatments were maintained for 8 weeks at which time the animals were sacrificed and joints assessed functionally, and joint tissue assessed at the histologic and the molecular levels (mRNA expression patterns). Treatment with ketotifen led to significantly improved function (less contraction) and a significant decline in nerves, 
mast cells and myofibroblasts in the capsular tissue [57]. Treatment also significantly modified the expression pattern for a number of relevant molecules in the capsular tissue [58]. Again, this outcome in another species added to the conclusion that use of mast cell stabilizers such as ketotifen in some abnormal healing situations could be of benefit to at risk patients who may experience joint contractures. In the above studies, the ketotifen was administered starting at the time of injury, so similar to the red Duroc pigs, additional studies where the administration of the ketotifen was delayed after injury may also provide insights in the "window of opportunity" to modify the contracture outcomes.

Currently, we are initiating a clinical trial of ketotifen in patients with elbow injuries (Hildebrand et al., in progress). Aligned with this trial is work to identify biomarkers for those at highest risk for developing joint contractures. As mentioned above, $\sim 15 \%$ of such patients develop such fibrotic joint contractures following a serious elbow injury, so it would be prudent to identify those at risk as early as possible to initiate appropriate treatment(s).

\subsection{Administration of Ketotifen to Scleroderma Patients}

In a number of studies [discussed in 59,60], elevated numbers of mast cells were detected in skin samples from involved skin of scleroderma patients. Many of the mast cells appeared histologically to be in a state of active degranulation. Other groups have also focused on mast cells in scleroderma patients, and suggested they may be a potential target for interventions [61]. Such findings, plus reports of ketotifen being effective in preventing skin thickening in the tsk/tsk mouse (the thick skinned mouse model of scleroderma) [62], led Gruber and Kaufman to use ketotifen in the treatment of a small number of patients with diffuse scleroderma [59]. Ultilizing a dose of $3 \mathrm{mg}$ ketotifen twice a day, it was found that the drug was effective in relieving some of the symptoms of scleroderma in two male patients with early diffuse disease. This small case report was followed by a larger 24 patient double-blind randomized control trial [60] of 6 months duration. Unfortunately, in this larger trial ketotifen was not found to be efficacious (using pulmonary function, global assessments and mast cell releasability), but some relief from pruritus was noted in some patients. In fact, the authors were unable to show mast cell suppression in their study. Why the initial two patients did receive some benefit from the ketotifen treatment, while those in the larger trial did not was likely due to variability in response patterns and perhaps a more advanced disease in some of the patients selected. Based on the porcine and rabbit studies discussed above regarding ketotifen use, one has to give the drug early after the injury/insult (skin wounding or joint injury). Thus, in scleroderma patients with established skin fibrosis ketotifen treatment may not be very effective due to the advanced state of the fibrotic response. Early scleroderma is known to be somewhat difficult to diagnose, so once skin involvement is very evident, it may be too late to have a strong impact using ketotifen to target mast cells. Likely it would be most effective during the edematous phase of the condition when initial mast involvement is just starting versus later when mast cells are still involved, but the fibrosis in the skin is established and inhibiting mast cells would possibly interfere with progression but not reverse established fibrosis (not unlike what we have observed in the rat bleomycin model discussed above when treated with urokinase).

While using ketotifen to address skin involvement in scleroderma has not yet been justified by the evidence, for a variety of reasons such as those discussed above, it might have application in patients that are progressing to pulmonary hypertension and pulmonary fibrosis, disease complications/phenotypes that may be detected early so intervention with ketotifen would potentially have a significant impact on this often fatal complication of the disease [discussed in 24]. Currently, evidence to support such a direction for use of ketotifen is meager, but we did have one patient with rapidly progressive scleroderma with pulmonary manifestations (fibrosis and pulmonary edema). The patient was treated with ketotifen on compassionate grounds after failing most other medical interventions. He rapidly exhibited some improvement in pulmonary function, was able to walk some distances, and regained some of his strength while on the drug. Unfortunately, he developed hives in relation to taking the drug and it was discontinued, and he died soon thereafter [discussed in 26]. Thus, in situations where pulmonary fibrosis becomes a feature of disease with something of a defined early starting point, ketotifen +/urokinase may be an effective treatment regimen for a subset of scleroderma patients evolving pulmonary involvement. In fact, one could envision adding tPA to a complex intervention regimen to address both vascular and pulmonary aspects of the condition. This speculation would certainly require a well designed clinical trial, but is an option to be entertained in the face of limited alternatives.

\subsection{Potential Application of Mast Cell Stabilizers $+/-$ Fibrinolytics in Other Human Fibrotic Conditions}

In addition to the above discussed conditions, other conditions or abnormal fibrotic response patterns in humans could also be envisioned to have efficacious treatments based on ketotifen and or fibrinolytics. These include hypertrophic scarring following thermal burn injuries, 
tendinopathies involving fibrosis, and possibly Dupuytren's contractures of the hand.

In burn patients, increasing size of the injury, or the location site increases the risk for hypertrophic scarring, an abnormal fibrogenic response [45,63]. Hypertrophic scars have abnormal collagen deposition, are hypercontracted due to myofibroblasts, have elevated numbers of mast cells and nerves, and can be itchy (pruritis), disfiguring, and if occurring across a joint, lead to joint contractures. Serum levels of histamine metabolites (histamine is a component of mast cells granules) are elevated in burn patients developing hypertrophic scars [64]. Many of these characteristics are similar to the pig and rabbit models discussed above where ketotifen was effective. Thus, ketotifen or other mast cell stabilizers should be considered for studies regarding effectiveness to prevent hypertrophic fibrosis development, or following revision surgery to prevent re-establishment of abnormal healing.

In tendon disorders such as Carpal Tunnel Syndrome (CTS), the initial phases of the condition involve a swelling/edema of the tendon sheath leading to fibrosis, pain, and compromised nerve function, often times requiring surgical interventions that are variable in outcomes [reviewed in 65; and others]. Abnormal collagen deposition is noted [66; and others], as well as the presence of mast cells and myofibroblasts but these cells have not been investigated for their role in the disease process. Mast cells have also been identified in other tendon disorders such as patellar tendinosis [67], but again their role has not been pursued. Interestingly, carpel tunnel-like syndromes have been reported in some scleroderma patients with early disease [discussed in 68], so there may be some common mechanisms of fibrosis operative here as well.

Dupuytren's contracture of the hand is a fibrotic process occurring in the fascia of the planar region which leads to a thickening of the fascia and loss of tendon function. This progressive condition appears to have a genetic component, and it is fairly common in some countries such as Sweden and Scandinavian countries [69-71]. Mast cells and nerves have been detected in the fibrotic tissues [72]. While the early stages of the process has not been studied in detail, the finding of mast cells, nerves and myofibroblasts certainly raise the possibility that agents such as ketotifen might have efficacy in preventing or inhibiting progression from the early phases. Presently, one approach to remove the deposited collagen is to treat the affected area with bacterial collagenase $[73,74]$ to degrade the fibrotic material. Whether such interventions have long lasting effects, or whether ketotifen could be used post-collagenase, remains to be studied.

It should be pointed out that ketotifen has been inves- tigated for use in rheumatoid arthritis (RA) where mast cells, nerves and fibrotic processes are known to occur in synovium and during pannus progression. Pannus progression occurs in RA due to chronic fibrin deposition (as well as attempts to remove it via endogenous fibrinolytic elements) with invasion of fibroblasts and collagen deposition. However, in clinical trials involving RA patients treated with ketotifen $+/-$ other drugs, ketotifen exhibited no detectable effects on disease parameters $[75,76]$. Therefore, unique disease features may influence whether mast cells stabilizers have efficacy in preventing the progression of fibrosis associated with some clinically defined conditions.

\section{SUMMARY}

From the above discussion, it is clear that fibrosis and fibrotic processes associated with specific conditions can be interfered with using fibrinolytic agents such as urokinase and tissue plasminogen activator. Such treatments are partially effective in both the lung and in the vasculature to mitigate chronic fibrosis where there is continual fibrin deposition contributing to the fibrosis. Thus, this approach alleviates the product of the process but does not stop the process itself. In contrast, the use of mast cell stabilizers is directed more upstream in the fibrotic process, and thus, work more effectively when administered early in the process. What has not been yet evaluated is the use of both approaches in the same models, or in selected patient populations. Thus, subsets of scleroderma patients may benefit from the tPA-focused vascular approach, while others with the condition and onset of pulmonary involvement may benefit from both urokinase + mast cell stabilizer protocols. A key element of all of these approaches is the ability to identify those patients who will benefit most (e.g. precision medicine) and conduct clinical trials to validate the appropriateness of the interventions.

While it is clear that other options are also potentially valuable in treating fibrotic conditions [e.g. controlling the expression and activity of pro-fibrotic growth factors such as TGF-betal and others; 77-79], the reagents and drugs discussed in this review are already approved for use in human populations and have extensive safety and efficacy data available regarding their use in humans. Thus, the application for the conditions described would potentially be new uses of existing reagents/drugs which would expedite their approval going forward.

\section{ACKNOWLEDGEMENTS}

The author thanks Drs. Marvin Fritzler and Kevin Hildebrand (McCaig Institute for Bone \& Joint Health) for many interesting discussions on these topics, and other colleagues, students and staff who worked on many of the projects discussed. The author also thanks Dr. Yamini Achari for her assistance in reviewing and editing the manuscript. The 
studies performed in the author's laboratory were supported by grants from The Arthritis Society, the Canadian Arthritis Network, and the Canadian Institutes for Health Research. The author declares no conflicts of interest, and none of the studies discussed were supported by industry other than to supply reagents to perform some of the studies (tPA and rh-urokinase).

\section{REFERENCES}

[1] Bagavant, H., et al. (2008) Lupus glomerulonephritis revisited 2004: Autoimmunity and end-organ damage. Scandinavian Journal of Immunology, 60, 52-63. doi:10.1111/j.0300-9475.2004.01463.x

[2] Krause, G., et al. (1992) LiCl prolongs survival and alters disease progression in the NZB/W model of SLE. Lithium, 3, 61-67.

[3] Hart, D.A., et al. (1994) Partial characterization of the enhanced survival of female NZB/W mice treated with lithium chloride. International Journal of Immunopharmacology, 16, 825-833. doi:10.1016/0192-0561(94)90056-6

[4] Lenz, S.P., Izui, S. and Hart, D.A. (1997) Evidence that lithium chloride treatment of female NZB/W mice does not influence autoantibody profiles in this murine model of systemic lupus erythematosus. Journal of Trace and Microprobe Techniques, 15, 109-116.

[5] Hart, D.A., et al. (1994) Partial reversal of established bleomycin-induced pulmonary fibrosis by rh-urokinase in a rat model. Clinical \& Investigative Medicine, 17, 69-76.

[6] Gharaee-Kertmani, M., et al. (2008) The role of urokinase in idiopathic pulmonary fibrosis and implication for therapy. Expert Opinion on Investigational Drugs, 17, 905-916. doi:10.1517/13543784.17.6.905

[7] Azambuja E., et al. (2005) Bleomycin lung toxicity: Who are the patients with increased risk? Pulmonary Pharmacology \& Therapeutics, 18, 363-366. doi:10.1016/j.pupt.2005.01.007

[8] Paun, A., et al. (2013) Association analysis reveals genetic variation altering bleomycin-induced pulmonary fibrosis in mice. American Journal of Respiratory Cell and Molecular Biology, 48, 330-336. doi:10.1165/rcmb.2012-00780C

[9] Scriabine, A. and Rabin, D.U. (2009) New developments in the therapy of pulmonary fibrosis. Advances in Pharmacology, 57, 419-464. doi:10.1016/S1054-3589(08)57011-6

[10] Mouratis, M.A. and Aidinis, V. (2011) Modeling pulmonary fibrosis with bleomycin. Current Opinion in Pulmonary Medicine, 17, 355-361. doi:10.1097/MCP.0b013e328349ac2b

[11] Chambers, R.C. (2008) Procoagulant signaling mechanisms in lung inflammation and fibrosis: Novel opportunities for pharmacological intervention? British Journal of Pharmacology, 153, S367-S378. doi:10.1038/sj.bjp.0707603

[12] Chambers, R.C. and Laurent, G.J. (2002) Coagulation cascade proteases and tissue fibrosis. Biochemical Society
Transactions, 30, 194-200. doi:10.1042/BST0300194

[13] Aksu, K., Donmez, A. and Keser, G. (2012) Inflammation-induced thrombosis: Mechanisms, disease associations and management. Current Pharmaceutical Design, 18, 1478-1493. doi:10.2174/138161212799504731

[14] Davalos, D. and Akassoplou, K. (2012) Fibrinogen as a key regulator of inflammation in disease. Seminars in Immunopathology, 34, 43-62. doi:10.1007/s00281-011-0290-8

[15] Ohba, T., et al. (1994) Scleroderma bronchoalveolar lavage fluid contains thrombin, a mediator of human lung fibroblast proliferation via induction of platelet-derived growth factor alpha-receptor. American Journal of Respiratory Cell and Molecular Biology, 10, 405-412. doi:10.1165/ajrcmb.10.4.7510986

[16] Imokawa, S., et al. (1997) Tissue factor expression and fibrin deposition in the lungs of patients with idiopathic pulmonary fibrosis and systemic sclerosis. American Journal of Respiratory and Critical Care Medicine, 156, 631-636. doi:10.1164/ajrccm.156.2.9608094

[17] Cash, H.A., et al., (1979) A rat model of chronic respiretory infection with Pseudomonas aeruginosa. American Review of Respiratory Disease, 119, 453-459.

[18] Vogt, S.L., et al. (2011) The stringent response is essential for Pseudomonas aeruginosa virulence in the rat lung agar bead and Drosophila melanogaster feeding models of infection. Infection and Immunity, 79, 4094-4104. doi:10.1128/IAI.00193-11

[19] Hart, D.A., et al. (1993) Exogenous rh-urokinase modifies inflammation and Pseudomonas aeruginosa infection in a rat chronic pulmonary infection model. Canadian Journal of Microbiology, 39, 1127-1134. doi: $10.1139 / \mathrm{m} 93-170$

[20] Hart, D.A. and Woods, D.E. (1994) Urokinase enhances the growth of Pseudomonas spp in vitro under nonshaking (oxygen limited) conditions. Canadian Journal of Microbiology, 40, 292-297. doi:10.1139/m94-047

[21] Hart, D.A. and Woods, D.E. (1994) Human urokinase, a serine proteinase, potentiates the in-vitro growth of microorganisms which commonly infect burn patients. Journal of Medical Microbiology, 41, 264-271. doi:10.1099/00222615-41-4-264

[22] Krieg, T. and Takehara, K. (2009) Skin disease: A cardinal feature of systemic sclerosis. Rheumatology (Oxford), 48, 14-18. doi:10.1093/rheumatology/kep 108

[23] Matucci-Cerinic, M., et al. (2009) The complexity of managing systemic sclerosis: Screening and diagnosis. Rheumatology (Oxford), 48, 8-13. doi:10.1093/rheumatology/ken482

[24] Bussone, G. and Mouthon, L. (2011) Interstitial lung disease in systemic sclerosis. Autoimmunity Reviews, 10, 248-255. doi:10.1016/j.autrev.2010.09.012

[25] Valentini, G., et al., (2013) Early systemic sclerosis: Marker autoantibodies and videocapillaroscopy patterns are each associated with distinct clinical, functional and cellular activation markers. Arthritis Research \& Therapy, 15, R63. (Epub ahead of print) doi:10.1186/ar4236

[26] Fritzler, M.J. and Hart, D.A. (1990) Prolonged improve- 
ment of Raynaud's phenomenon and scleroderma after recombinant tissue plasminogen activator therapy. Arthritis \& Rheumatism, 33, 274-276. doi:10.1002/art.1780330218

[27] Wilson, D., et al. (1995) The safety and efficacy of lowdose tissue plasminogen activator in the treatment of systemic sclerosis. The Journal of Dermatology, 22, 637642.

[28] Lotti, T., Campanile, G. and Matucci-Cerinic, M. (1994) Cutaneous and plasma fibrinolytic activities are not deficient in patients with systemic sclerosis. Journal of the American Academy of Dermatology, 30, 813-814. doi:10.1016/S0190-9622(08)81531-9

[29] Ames, P.R., et al. (1997) The coagulation/fibrinolysis balance in systemic sclerosis: evidence for a haematological stress syndrome. British Journal of Rheumatology, 36, 1045-1050. doi:10.1093/rheumatology/36.10.1045

[30] Herrick, A.L., et al. (1996) Von willebrand factor, thrombomodulin, thromboxane, beta-thromboglobin and markers of fibrinolysis in primary Raynaud's phenomenon and systemic sclerosis. Annals of the Rheumatic Diseases, 55, 122-127. doi:10.1136/ard.55.2.122

[31] Bandinelli, F, et al. (2005) The fibrinolytic system components are increased in systemic sclerosis and modulated by Alprotadil (alpha1 ciclodestryn). Clinical and Experimental Rheumatology, 23, 671-677.

[32] Shimizu, Y., et al. (1994) Neural blockade, urokinase and prostaglandin E1 combination therapy for acute digital ischemia of progressive systemic sclerosis. The Journal of Dermatology, 21, 755-759.

[33] Ciompi, M.L., et al. (1996) A placebo-controlled study of urokinase therapy in systemic sclerosis. Biomedicine \& Pharmacotherapy, 50, 363-368. doi:10.1016/S0753-3322(96)89669-7

[34] Bazzichi, L., et al. (2003) Clinical improvement in systemic sclerosis resulting from urokinase therapy explained by light and electron microscopy skin examination. Scandinavian Journal of Rheumatology, 32, 261-267. doi:10.1080/03009740310003875

[35] Klimiuk, P.S., et al. (1992) A double blind placebo controlled trial of recombinant tissue plasminogen activator in the treatment of digital ischemia in systemic sclerosis. The Journal of Rheumatology, 19, 716-720.

[36] Fritzler, M.J., Hart, D.A., et al. (1995) Antibodies to fibrin bound tissue type plasminogen activator in systemic sclerosis. The Journal of Rheumatology, 22, 1688-1693.

[37] Kessler-Becker, D., et al. (2004) High plasminogen activator inhibitor type 2 expression is a hallmark of scleroderma fibroblasts in vitro. Experimental Dermatology, 13, 708-714. doi:10.1111/j.0906-6705.2004.00222.x

[38] Postiglione, L., et al. (2010) The plasminogen activator system in fibroblasts from systemic sclerosis. International Journal of Immunopathology and Pharmacology, 23, 891-900.

[39] Manetti, M., et al. (2011) A genetic variation located in the promoter region of the UPAR (CD87) gene is associated with the vascular complications of systemic sclerosis. Arthritis \& Rheumatism, 63, 247-256. doi:10.1002/art.30101

[40] Gallant, C.L., Olson, M.E. and Hart, D.A. (2004) Molecular, histologic, and gross phenotype of skin wound healing in red Duroc pigs reveals an abnormal healing phenotype of hypercontracted, hyperpigmented scarring. Wound Repair and Regeneration, 12, 305-319. doi:10.1111/j.1067-1927.2004.012311.x

[41] Gallant-Behm, C.L. and Hart, D.A. (2006) Genetic analysis of skin wound healing and scarring in a porcine model. Wound Repair and Regeneration, 14, 46-54. doi:10.1111/j.1524-475X.2005.00087.x

[42] Gallant-Behm, C.L, Olson, M.E. and Hart, D.A. (2005) Cytokine and growth factor mRNA expression patterns associated with the hypercontracted, hyperpigmented healing phenotype of red Duroc pigs: A model of abnormal human scar development? Journal of Cutaneous Medicine and Surgery, 9, 165-177. doi:10.1007/s10227-005-0105-4

[43] Gallant-Behm, C.L., et al. (2007) Genetic involvement in skin wound healing and scarring in domestic pigs: Assessment of molecular expression patterns in (Yorkshire $\mathrm{x}$ Red Duroc) x Yorkshire backcross animals. Journal of Investigative Dermatology, 127, 233-244. doi:10.1038/sj.jid.5700482

[44] Zhu, K.Q., et al. (2007) Review of the female Duroc /Yorkshire pig model of human fibroproliferative scarring. Wound Repair and Regeneration, 17, S32-S39. doi:10.1111/j.1524-475X.2007.00223.x

[45] Engrav, L.H., Garner, W.L. and Tredget, E.E. (2007) Hypertrophic scar, wound contraction and hyper-pigmentation. Journal of Burn Care \& Research, 28, 593-597. doi:10.1097/BCR.0B013E318093E482

[46] Gallant-Behm, C.L., Hildebrand, K.A. and Hart, D.A. (2008) The mast cell stabilizer ketotifen prevents development of excessive skin wound contraction and fibrosis in red Duroc pigs. Wound Repair and Regeneration, 16, 226-233. doi:10.1111/j.1524-475X.2008.00363.x

[47] de Hemptinne, I., et al. (2008) Dermal fibroblasts from red Duroc and Yorkshire pigs exhibit intrinsic differences in the contraction of collagen gels. Wound Repair and Regeneration, 16, 132-142. doi:10.1111/j.1524-475X.2007.00340.x

[48] Ohtola, J., et al. (2008) Beta-catenin has sequential roles in the survival and specification of ventral dermis. Development, 135, 2321-2329. doi:10.1242/dev.021170

[49] Hildebrand, K.A., et al. (2004) Myofibroblast numbers are elevated in human elbow contractures after trauma. Clinical Orthopaedics and Related Research, 419, 189. 197.

[50] Hildebrand, K.A., Zhang, M. and Hart, D.A. (2005) High rate of joint capsule matrix turnover in chronic human elbow contractures. Clinical Orthopaedics and Related Research, 439, 228-234.

[51] Hildebrand, K.A., Zhang, M. and Hart, D.A. (2007) Myofibroblast upregulators are elevated in joint capsules in posttraumatic contractures. Clinical Orthopaedics and Related Research, 456, 85-91.

[52] Lindenhovius, A.L. and Jupiter, J.B. (2007) The post- 
traumatic stiff elbow: A review of the literature. Journal of Hand Surgery, 32, 1605-1623. doi:10.1016/j.jhsa.2007.09.015

[53] Charalambous, C.P. and Morrey, B.E. (2012) Posttraumatic elbow stiffness. The Journal of Bone \& Joint Surgery, 94, 1428-1437. doi:10.2106/JBJS.K.00711

[54] Hildebrand, K.A., Zhang, M. and Hart, D.A. (2006) Joint capsule matrix turnover in a rabbit model of chronic joint contractures: Correlation with human contractures. Journal of Orthopaedic Research, 24, 1036-1043. doi:10.1002/jor.20128

[55] Hildebrand, K.A., et al. (2008) Cellular, matrix, and growth factor components of the joint capsule are modified early in the process of posttraumatic contracture formation in a rabbit model. Acta Orthopaedica, 79, 116125. doi:10.1080/17453670710014860

[56] Hildebrand, K.A., et al. (2008) Joint capsule mast cells and neuropeptides are increased within four weeks of injury and remain elevated in chronic stages of posttraumatic contractures. Journal of Orthopaedic Research, 26, 1313-1319. doi:10.1002/jor.20652

[57] Monument, M.J., Hart, D.A., et al. (2010) The mast cell stabilizer ketotifen fumarate lessens contracture severity and myofibroblast hyperplasia: A study in a rabbit model of posttraumatic joint contractures. The Journal of Bone \& Joint Surgery, 92, 1468-1477. doi:10.2106/JBJS.I.00684

[58] Monument, M.J., Hart, D.A., et al. (2012) The mast cell stabilizer ketotifen reduces joint capsule fibrosis in a rabbit model of post-traumatic contractures. Inflammation Research, 61, 285-292. doi:10.1007/s00011-011-0409-3

[59] Gruber, B.L. and Kaufman, L.D. (1990) Ketotifen-induced remission of progressive early diffuse scleroderma: Evidence for the role of mast cells in disease pathogenesis. The American Journal of Medicine, 89, 392-395. doi:10.1016/0002-9343(90)90360-P

[60] Gruber, B.L. and Kaufman, L.D. (1991) A double-blind randomized controlled trial of ketotifen versus placebo in early diffuse scleroderma. Arthritis \& Rheumatism, 34, 362-366. doi:10.1002/art.1780340315

[61] Yukawa, S., et al. (2010) Involvement of mast cells in systemic sclerosis. Nihon Rinsho Meneki Gakkai Kaishi, 33, 81-86. doi: $10.2177 /$ jsci.33.81

[62] Walker, M., Harley, R. and LeRoy, E.C. (1990) Ketotifen prevents skin fibrosis in the tight skin mouse. The Journal of Rheumatology, 17, 57-59.

[63] Zhu, Z., et al. (2013) The molecular mechanism of hypertrophic scar. Journal of Cell Communication and Signaling. Epub ahead of print.

[64] Tredget, E.E., et al. (1997) Determination of plasma $\mathrm{N}^{\mathrm{T}}$-methylhistamine in vivo by isotope dilution using benchtop gas chromatography-mass spectrometry. Sciences and Applications, 694, 1-9. doi:10.1016/S0378-4347(97)00122-9

[65] Wilder-Smith, E.P., Seet, R.C. and Lim, E.C. (2006) Diagnosing carpel tunnel syndrome-clinical criteria and ancillary tests. Nature Clinical Practice. Neurology, 2, 366374.
[66] Kuhnel, W., et al. (1987) A morphological study of the peri- and epineurium in the compression zone of the median nerve in carpal tunnel syndrome. Acta Anatomica (Basal), 129, 81-91.

[67] Scott, A., et al. (2008) Increased mast cell numbers in human patellar tendinosis: Correlation with symptom duration and vascular hyperplasia. British Journal of Sports Medicine, 42, 753-757. doi:10.1136/bjsm.2007.040212

[68] Medsger Jr., T.A. (2003) Natural history of systemic sclerosis and the assessment of disease activity, severity, functional status, and psychologic well being. Rheumatic Disease Clinics of North America, 29, 255-273. doi:10.1016/S0889-857X(03)00023-1

[69] Murrell, G.A., Francis, M.J. and Howlett, C.R. (1989) Dupuytren's contracture. Fine structure in relation to etiology. Journal of Bone and Joint Surgery British, 71, 367373.

[70] Dolmans, G.H., de Bock, G.H. and Werker, P.M. (2012) Dupuytren diathesis and genetic risk. Journal of Hand Surgery, 37, 2106-2111. doi:10.1016/j.jhsa.2012.07.017

[71] Hu, F.Z., et al. (2005) Mapping of an autosomal dominant gene for Dupuytren's contracture to chromosome $16 \mathrm{q}$ in a Swedish family. Clinical Genetics, 68, 424-429. doi:10.1111/j.1399-0004.2005.00504.x

[72] Shubert, T.E., et al. (2006) Dupuytren's contracture is associated with sprouting of substance $P$ positive nerve fibres and infiltration by mast cells. Annals of the Rheumatic Diseases, 65, 414-415. doi:10.1136/ard.2005.044016

[73] Azzopardi, E. and Boyce, D.E. (2012) Clostridium histolyticum collagenase in the treatment of Dupuytren's contracture. British Journal of Hospital Medicine (London), 73, 432-436.

[74] Hentz, V.R., et al. (2012) Advances in the management of Dupuytren's disease: Collagenase. Hand Clinics, 28, 551-563. doi:10.1016/j.hcl.2012.08.003

[75] Pullar, T. and Capell, H.A. (1985) A rheumatological dilemma: Is it possible to modify the course of rheumatoid arthritis? Can we answer the question? Annals of the Rheumatic Diseases, 44, 134-140. doi:10.1136/ard.44.2.134

[76] Teh, L.G., Madhok, R. and Capell, H.A. (1984) Does the addition of ketotifen to non-steroidal anti-inflammatory drugs confer and additional benefit to rheumatoid arthritis? British Journal of Clinical Pharmacology, 17, 157-159. doi:10.1111/j.1365-2125.1984.tb02330.x

[77] Kolb, M., et al. (2001) Proteoglycans decorin and biglycan differentially modulate TGF-beta-mediated fibrotic responses in the lung. American Journal of Physiology. Lung Cellular and Molecular Physiology, 280, L1327L1334.

[78] Gagliardini, E. and Benigni, A. (2006) Role of anti-TGFbeta antibodies in the treatment of renal injury. Cytokine \& Growth Factor Reviews, 17, 89-96. doi:10.1016/i.cytogfr.2005.09.005

[79] Boffa, J.J. and Ronco, P. (2007) Strategies to reverse fibrotic lesions of the kidney. La Presse Médicale, 26, 1857-1864. doi:10.1016/j.lpm.2007.04.033 